quite superficial. However, after GHAE, one-week training courses on disaster medicine are held regularly at the National Hospital Tokyo Disaster Medical Center, and realistic disaster drills are adopted and performed mainly in disaster core hospitals.

The establishment of Disaster Medical Assistant Teams (DMATs), composed by emergency physicians, nurses, and emergency medical technicians, has been a long-time dream in Japan. In August 2004, thanks to the efforts of the people concerned, DMATs were organized in Tokyo. This new effort is expected to expand into other areas in the near future. Several other improved areas and their problems are discussed in the presentation.

Keywords: anniversary; assessment; core hospitals; disaster medical assistance teams (DMATs); disaster medicine; education; exercises; Great Hanshin-Awaji Earthquake (GHAE); Hyogo Prefecture; Japan

Prebosp Disast Med 2005;20(3):s126-s127

\section{Hospital Preparedness for Disasters}

Felipe Cruz Vega

Pan-American Association for Emergency and Disaster Medicine, Mexico

The Pan-American Health Organization (PAHO) and the Mexican Federal Government signed an agreement to establish the voluntary and temporary certification at the institutional, national, and international levels of health installations prepared to face disaster situations.

The Mexican Social Security Institute was interested in this initiative, taking into account the number of hospitals that form its infrastructure: 254 hospitals of medium and high complexity, of which 131 (52\%) are situated in highrisk areas, $67(26 \%)$ in medium-risk areas, and only 56 $(22 \%)$ in low-risk areas.

Because of the aforementioned statistics, a model was designed to certify the hospitals in order to establish a permanent and priority program entitled "Hospitals Prepared to Face Disaster Situations" in agreement with the PAHO recommendations. The Institute, through the Institutional Committee for Disaster Cases, created rules for the creation of the "Hospital Plan for Disaster Cases", which includes structural and non-structural aspects of the organization and specified the governing body for each organization.

Each hospital unit is responsible for designing its own plan, which should designate the actions to be followed in an internal or external disaster in the stages before, during, and after the event, including identifying the risk factors, vulnerability, human resources, and materials at their disposal ("made to measure").

For the certification stage, the Committee designed an "instrument" for quantitative evaluation, which permits the qualification of the medical units to select the hospitals classified as high-resolution level and situated in high-risk areas.

Later assessment visits were programmed and the evaluation procedure was applied. Forty hospitals have achieved this Certification, and others currently are in the process.

At the present time, the Institute is involved in the national level certification stage. This certification will ensure the security of users and installations, and will facilitate a decrease in insurance premiums, which will be reinvested in strengthening hospital security.

Keywords: certification; coordination; disaster; evaluation; hospital; Mexico; preparedness

Prebosp Disast Med 2005;20(3):s127

\section{Australian Mass-Casualty, Burn, Disaster Plan F. Wood, ${ }^{1}$ D. Cooper, ${ }^{2}$ A. Robertson, ${ }^{3}$ D. Edgar ${ }^{3}$ \\ 1. Royal Perth Hospital, Australia \\ 2. Ambulance Service of NSW, Australia \\ 3. Health Department of WA, Australia}

Australia has a series of dedicated burn units. Emergency medial services in capital cities respond to the emergency needs of the population of 20 million. The significant distance involved in transporting patients necessitates a twophase response to a mass-casualty event: (1) The surge phase is the initial phase of rapid expansion of urgent services provided by core health services of the given state or territories; and (2) The redistribution phase is the protracted period of ongoing needs for medical care that challenges the capacity of the given state or territory due to the prolonged healing and rehabilitation needs of burn survivors.

We intend to develop a coordinated national response for events involving multiple burn casualties. The plan will be based on the international literature in context of local resources and conditions. The plan will interface with the disaster plan of the states and territories in addition to the overseas mass-casualty plans of the Australian Government. The development of the two-phase, kinetic, mass-disaster model is an original concept that provides a template for any mass-casualty event in Australia or overseas. This plan will be discussed.

Keywords: burns; coordination; disaster; mass casualties; phases; planning; response Prebosp Disast Med 2005;20(3):s127

\section{Shipping Disasters in the English Channel: A Need for International, Multidisciplinary Rescue $K$. Vandevelde Belgium}

On 06 March 1987, the Herald of Free Enterprise (HOFE), a car ferry transporting 543 passengers and crew, 42 trucks, and 84 cars, capsized one mile away from Zeebrugge harbor. A large rescue operation began at three operational levels. Rudimentary means of rescue were attempted on-board the wreck. Helicopters transported victims from the wreck to a nearby military harbor, and boats were directed towards an empty pontoon. At the pontoon, emergency care was provided and further transport was organized to surrounding hospitals. The available resources made it possible to start advanced life support (ALS) at the triage station, where 21 medical teams received $>250$ victims within hours after the event. The majority of casualties were due to immersion, while most of the injuries were minor orthopedic trauma, bruises, and cuts, which could be treated easily. A few victims with cardiac arrest and hypothermia were referred to a hospital for 